\title{
Reduction of ventricular size after shunting for normal pressure hydrocephalus related to CSF dynamics before shunting
}

\author{
J Th J TANS, D C J POORTVLIET
}

From the Department of Neurology and the Research Unit for Clinical Neurophysiology MBL-TNO, Westeinde Hospital, The Hague, The Netherlands

SUMMARY Reduction of ventricular size was determined by repeated computed tomography in 30 adult patients shunted for normal pressure hydrocephalus (NPH) and related to the pressurevolume index (PVI) and resistance to outflow of cerebrospinal fluid (Rcsf) measured before shunting. Rapid and marked reduction of ventricular size $(n=10)$ was associated with a significantly lower PVI than slow and moderate to marked $(n=13)$ or minimal to mild reduction $(n=7)$. Otherwise no relationship could be found between the reduction of ventricular size and PVI or Rcsf. It is concluded that both rate and magnitude of reduction of ventricular size after shunting for NPH are extremely variable. High brain elasticity seems to be the best predictor of rapid and marked reduction.

Reduction of ventricular size after shunting for normal pressure hydrocephalus (NPH) is reportedly not related to clinical improvement. ${ }^{1-4}$ The most reliable method for prediction of the result of shunting is determination of the resistance to outflow of cerebrospinal fluid (Rcsf) by means of constant flow or constant pressure infusion techniques. ${ }^{5-7}$ This Rcsf also appeared to be independent of ventricular size both before and after shunting. ${ }^{89}$ A second major parameter affecting CSF dynamics is the volumepressure relationship, which can be expressed as the pressure-volume index (PVI). A poor correlation was found between PVI and preoperative ventricular size $e^{9-11}$ but the relationship with postoperative ventricular dimensions has not been investigated. Since the PVI reflects brain elasticity one might hypothesise that reduction of ventricular size will be greater in patients with greater elasticity, whereas dilated ventricles surrounded by a compliant brain are less likely to diminish in size.

In some patients reduction of ventricular size is completed within days or weeks, whereas in others ventricular size decreases slowly over a period of

Address for reprint requests: Dr J Th J Tans, Department of Neurology, Westeinde Hospital, PO Box 432, 2501 CK The Hague, The Netherlands.

Received 29 May 1987 and in revised form 17 September 1987. Accepted 17 October 1987 months or even years. Surprisingly the rate of reduction has seldom been considered by previous investigators.

The aim of the present study was to collect data on both the magnitude and the rate of changes in ventricular size and to relate these to PVI and Rcsf.

\section{Material and methods}

We selected 30 adult patients with NPH who met the following criteria: (1) A cerebrospinal fluid (CSF) baseline pressure $<15 \mathrm{mmHg}$ and a $\mathrm{Rcsf}>13 \mathrm{mmHg} / \mathrm{ml} / \mathrm{min}$. (2) Insertion of a ventriculo-atrial shunt with medium pressure Holter valve. (3) No complications during the follow-up period. (4) No accompanying neurological conditions. (5) At least one preoperative and two postoperative computed tomograms (CT) with adequate visualisation of the ventricular system.

CSF pressure was measured by means of a ventricular catheter or via the lumbar route. Rcsf was obtained by constant flow infusion at a rate of 1.6 or $0.82 \mathrm{ml} / \mathrm{min}$. In addition a series of at least three bolus infusions of the same or different volumes was administered; from these data one PVI was calculated. ${ }^{56}$

CT scans of all patients were obtained within a 3-month period before surgery as well as one month and 2 to 24 months after surgery. A fourth CT scan was obtained in 21 , a fifth in 6 and a sixth in 2 cases, making a total of 119 CT scans. Using a translucent millimeter ruler ventricular and skull sizes were measured from the $C T$ images and expressed as the following ventricular index: $(A+B+3 C+E) / D$, in which $A$ is the maximum distance between the frontal tips, $B$ the maximum diameter of the lateral ventricles at the level of 
Table 1 Ventricular indices before and after shunting

\begin{tabular}{|c|c|c|c|c|c|}
\hline & \multirow{2}{*}{$\begin{array}{l}\text { No of } \\
\text { examinations }\end{array}$} & \multirow{2}{*}{$\begin{array}{l}\text { Interval } \\
\text { surgery-CT } \\
\text { months }\end{array}$} & \multicolumn{3}{|c|}{ Ventricular Index } \\
\hline & & & mean & $S D$ & range \\
\hline $\begin{array}{l}\text { Preoperative CT } \\
\text { 1st follow-up CT } \\
\text { 2nd follow-up CT } \\
\text { 3rd follow-up CT } \\
\text { 4th follow-up CT } \\
\text { 5th follow-up CT }\end{array}$ & $\begin{array}{r}30 \\
30 \\
30 \\
21 \\
6 \\
2\end{array}$ & $\begin{array}{c}\overline{0} \cdot 5 \pm 0 \cdot 2 \\
8 \cdot 0 \pm 5 \cdot 8 \\
19 \cdot 3 \pm 14 \cdot 1 \\
35 \cdot 0 \pm 20 \cdot 9 \\
67 \cdot 6 \pm 38 \cdot 3\end{array}$ & $\begin{array}{r}122 \cdot 9 \\
94 \cdot 5 \\
73 \cdot 7 \\
76 \cdot 3 \\
60 \cdot 3 \\
45 \cdot 5\end{array}$ & $\begin{array}{l}21 \cdot 7 \\
30 \cdot 1 \\
27 \cdot 8 \\
29 \cdot 1 \\
22 \cdot 5 \\
12 \cdot 0\end{array}$ & $\begin{array}{l}83-171 \\
46-164 \\
46-150 \\
37-150 \\
37-89 \\
37-54\end{array}$ \\
\hline
\end{tabular}

the caudate nucleus, $\mathrm{C}$ the maximum width of the third ventricle, $\mathrm{D}$ the maximum external diameter of the temporal bone and $\mathrm{E}$ the maximum distance between the cella mediae (fig 1).

The reduction of ventricular size was determined by calculating reduction ratios $(R R)$ :

$$
R_{1} \frac{\text { preoperative ventricular index }}{\text { ventricular index at 1st follow-up CT }} .
$$

The maximum $R R\left(R_{m}\right)$ was obtained by dividing the preoperative by the lowest postoperative ventricular index.

For patients with no or only a limited alteration in ventricle size and without clinical improvement, shunt malfunction was ruled out by a repeat constant flow infusion test.

\section{Results}

The results of the ventricular measurements are summarised in tables 1 and 2 . The largest reduction of ventricular size was obtained within the first month, but considerable reductions were found thereafter. The mean ventricular index of the third follow-up CT was higher than that of the second because more CT scans were performed in patients with mild to moderate reductions. The $R_{\mathrm{m}}$ was derived from CT scans performed a mean period of 12.4 months after surgery, illustrating the slow normalisation of ventricular dimensions.

No correlation could be found between the reduction of ventricular size $\left(R R_{m}\right)$ and the parameters of CSF dynamics, (PVI and Rcsf). Linear regression analysis yielded coefficients of correlation as low as
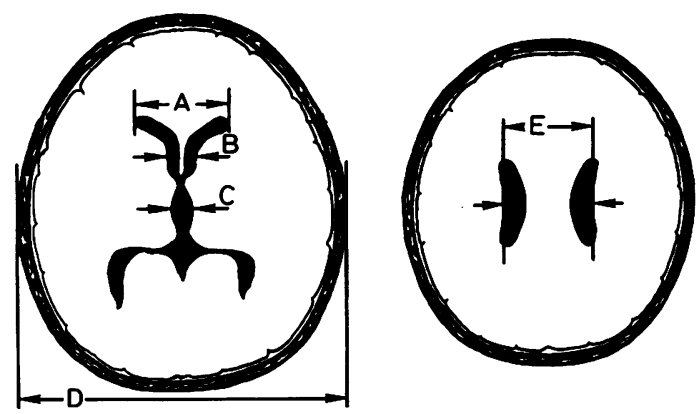

Fig 1 Ventricular and skull sizes. Ventricular index $=(A+B+3 C+E) / D$.
-0.29 and -0.01 . It is also important to note that no relationship could be established between preoperative ventricular index and PVI, Rcsf or $\mathbf{R R}_{\mathrm{m}}$. Therefore, ventricular dimensions before and after shunting seem to be independent of disturbances of CSF dynamics.

Analysing again the results of the ventricular measurements and taking into account both the magnitude and the rate of reduction three patterns could be distinguished:

(1) A rapid and marked reduction of ventricular size followed by a stable or slightly decreasing ventricular volume (fig $2 a$ ). In this group maximum or almost maximum reduction was achieved within one month in seven of the 10 cases and within 2.5 months in the remaining three. The mean $R_{1}$ was high compared with that of groups 2 and 3 the $R_{\mathrm{m}}$ too was high in group 1 , intermediate in group 2 and low in group 3 (table 3 ).

(2) A slow and moderate to marked reduction of ventricular size (fig $2 b$ ). After a limited decrease in the first month, ventricular volume continued to decrease during the first year and even during the second year in a number of cases. The smallest ventricles in these 13 patients were measured after a mean of $18.1 \pm 14.9$ months. The course of the reduction, as depicted in fig 2 , depended among other things on the number of and time interval between CT examinations. Determination of the true reduction rate requires a prospective study with serial CT scanning at regular times after surgery. However, the three different patterns of ventricular reduction are clearly demonstrated in fig 2 and table 3.

(3) A minimal to mild change in ventricle size with a $R R_{m}$ of less than 135 (fig 2c, table 3). Six of the seven patients of this group showed a small initial

Table 2 First, second and maximum reduction ratios

\begin{tabular}{lllll}
\hline & & \multicolumn{3}{l}{ Reduction ratio } \\
\cline { 3 - 5 } & $\begin{array}{l}\text { No of } \\
\text { examinations }\end{array}$ & mean & $S D$ & range \\
\hline$R_{R_{1}}$ & 30 & $141 \cdot 1$ & $48 \cdot 4$ & $100-274$ \\
$\mathbf{R R}_{2}$ & 30 & $183 \cdot 2$ & $56 \cdot 4$ & $100-283$ \\
$\mathbf{R R}_{\mathrm{m}}$ & 30 & $194 \cdot 4$ & $62 \cdot 0$ & $112-356$ \\
\hline
\end{tabular}



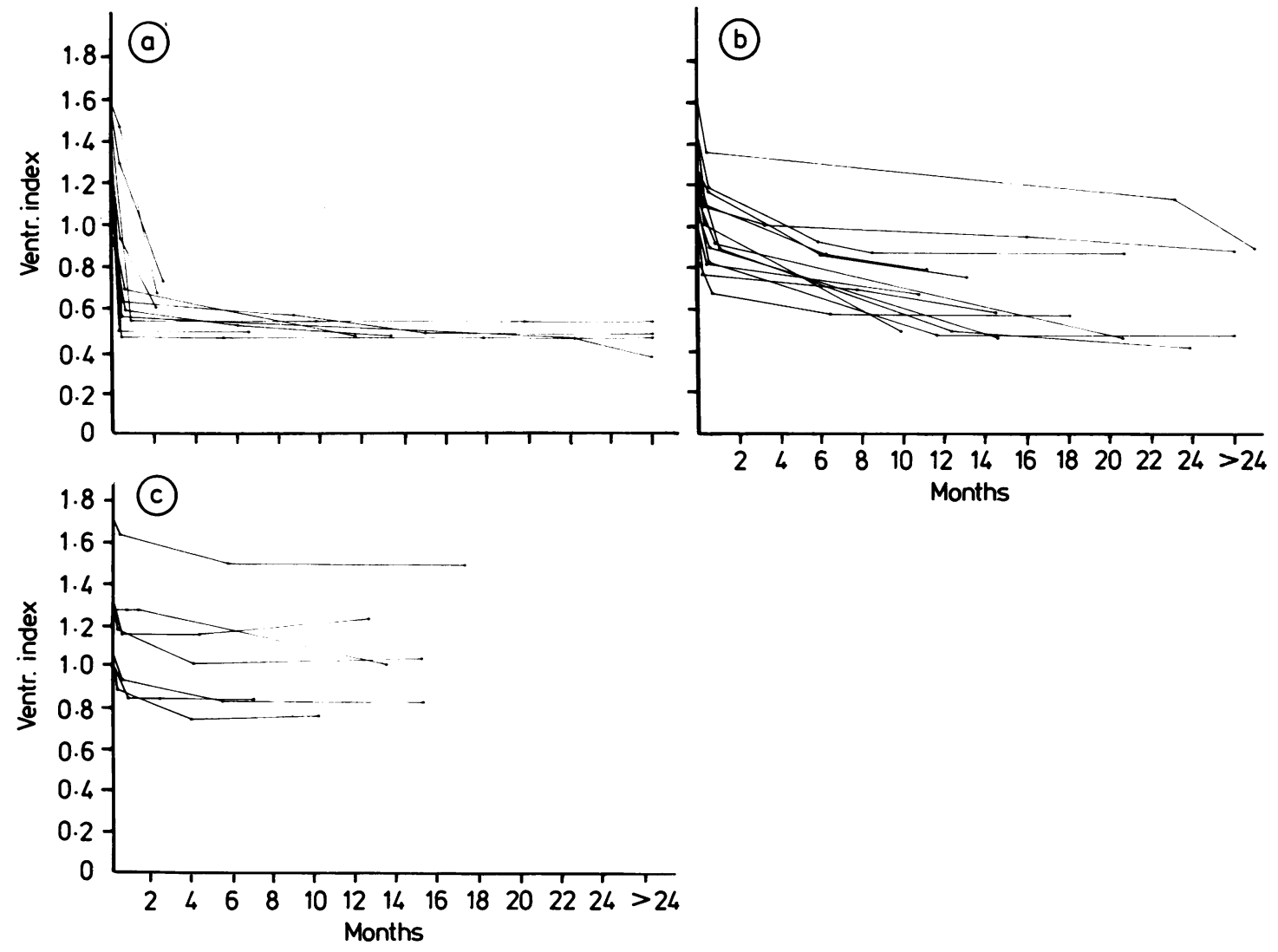

Fig 2 Ventricular index before (on y-axis) and after shunting (a) Group 1, $n=10$ : rapid and marked reduction of ventricular size. (b) Group 2, $n=13:$ slow and moderate to marked reduction. (c) Group 3, $n=7:$ minimal to mild reduction.

reduction followed by a further slight decrease or a minimal increase of ventricular size. Since the 7 th patient exhibited some secondary ventricular diminution, none of the 30 patients had a totally unchanged ventricular system.

The patients of group 1 exhibited a low PVI indicating tight brain conditions, whereas the PVI of group 2 was only mildly decreased and that of group 3 was almost within normal limits $(>18 \mathrm{ml})$. Applying analysis of variance the differences between group 1 and the others attained statistical significance (table 3). Such a difference did not emerge for Rcsf although the mean Rcsf of group 1 was higher than that of groups 2 and 3. Non-parametric analysis yielded very similar results.

Interestingly, PVI for those patients of group 2 who presented with a markedly increased Rcsf $(>18 \mathrm{mmHg} / \mathrm{ml} / \mathrm{min}$ ) was within the normal range, suggesting that the comparatively low brain elasticity was responsible for the slow rate of change of the ventricles. In contrast, the patients of group 3 exhibited an inverse relationship between Rcsf and PVI.

Table 3 Pattern of ventricular reduction related to reduction ratios, PVI and Rcsf

\begin{tabular}{|c|c|c|c|c|c|c|c|c|c|c|}
\hline & \multicolumn{3}{|c|}{$\begin{array}{l}\text { Group } 1 \\
n=10\end{array}$} & \multicolumn{3}{|c|}{$\begin{array}{l}\text { Group } 2 \\
n=13\end{array}$} & \multicolumn{3}{|c|}{$\begin{array}{l}\text { Group } 3 \\
n=7\end{array}$} & \multirow[b]{2}{*}{$P$} \\
\hline & mean & $S D$ & range & mean & $S D$ & range & mean & $S D$ & range & \\
\hline $\begin{array}{l}\mathrm{RR}_{1} \\
\mathrm{RR}_{\mathrm{m}} \\
\mathrm{PVI}^{\mathrm{R}} \\
\mathrm{Rcsf}\end{array}$ & $\begin{array}{r}187 \cdot 4 \\
247 \cdot 1 \\
12 \cdot 3 \\
24 \cdot 3\end{array}$ & $\begin{array}{r}61 \cdot 4 \\
45 \cdot 2 \\
2 \cdot 7 \\
8 \cdot 6\end{array}$ & $\begin{array}{r}107-252 \\
202-356 \\
9 \cdot 4-16 \cdot 6 \\
14 \cdot 4-35 \cdot 7\end{array}$ & $\begin{array}{r}122.4 \\
192.7 \\
16.0 \\
19.5\end{array}$ & $\begin{array}{r}8 \cdot 0 \\
48 \cdot 5 \\
3.7 \\
6.5\end{array}$ & $\begin{array}{c}109-142 \\
142-300 \\
9 \cdot 3-20 \cdot 6 \\
13 \cdot 1-37 \cdot 8\end{array}$ & $\begin{array}{r}109 \cdot 6 \\
122 \cdot 0 \\
17 \cdot 7 \\
20 \cdot 6\end{array}$ & $\begin{array}{l}5 \cdot 9 \\
7 \cdot 6 \\
5 \cdot 1 \\
6 \cdot 3\end{array}$ & $\begin{array}{l}100-118 \\
112-133 \\
12 \cdot 0-24 \cdot 9 \\
13 \cdot 8-31 \cdot 7\end{array}$ & $\begin{array}{l}0.0001 \\
0.0001 \\
0.02 \\
0.29\end{array}$ \\
\hline
\end{tabular}


Since groups 2 and 3 differed mainly in the magnitude of ventricular reduction and their PVIs were fairly similar we infer that brain elasticity has much less influence on the magnitude than on the rate of decline in ventricular size.

The question remains why the ventricular system failed to reduce in the seven patients of group 3. Three of these patients had a mildly increased Rcsf accompanied by a high PVI. Since they presented with a long history and exhibited no or only marginal improvement after shunting, they have to be categorised as cases of burnt out or compensated NPH. Two patients fulfilled all clinical, radiological and CSF dynamical criteria for NPH but the function of the shunt remained doubtful. Despite a normal infusion test, a new shunt was recently inserted in one patient, resulting in both clinical improvement and ventricular reduction. Finally two patients demonstrated the well known but unexplained combination of markedly disturbed CSF dynamics, a good to excellent clinical result and an almost unchanged ventricular system.

\section{Discussion}

Linear measurements of CT images of the ventricular system provide sufficiently reliable results to dispense with the more complicated planimetric and volumetric methods. ${ }^{12}$ Because NPH is associated with global ventricular enlargement, ventricular dimensions were assessed at four different sites. The sum of the maximum distance between the frontal tips and the caudate nucleus is the Huckman number that has been shown to correlate well with the area of the anterior horn. ${ }^{13}$ The maximum diameter of both cella mediae, representing the body of the lateral ventricles is assessed by most investigators who use multiple linear measurements. ${ }^{12-14}$ The width of the third ventricle was included because its walls perpendicular to the plane of the CT section and midsagittal localisation permit measurement that is subject to less error. ${ }^{15}$ In order to have one value for the ventricular size all measurements were incorporated in one ventricular index. The width of the third ventricle was weighted by a factor 3 so that it would be comparable to the other ventricular dimensions.

The presence of a well functioning ventriculoatrial shunt with a medium pressure Holter valve was a criterion for inclusion in the study. However, the opening pressure measured at surgery varied from 60 to $100 \mathrm{~mm}$ water and after insertion shunt function was measured only in patients suspected of malfunction. The hydrodynamic properties of CSF shunt systems were investigated in vitro. Slit type valves revealed variable opening and closing pressures and the flow tended to decrease with time. ${ }^{16}$ For a given differential pressure the flow rate depends on the re- sistance of the valve and that is not known. ${ }^{17}$ Therefore shunt characteristics may have influenced the process of reduction of ventricular size. In one study $79 \%$ of patients with NPH receiving a low pressure shunt showed ventricular reduction compared with $40 \%$ of those with a medium pressure shunt. ${ }^{15}$

Reduction of ventricular size after shunting for NPH is usually assessed within 6 to 12 months after surgery. In the present study substantial reductions were observed after that period. Therefore studies relating reduction of ventricular size to clinical improvement or other parameters should include CT scans taken at longer intervals after surgery, at least for those patients who do not exhibit rapid and marked reduction.

The lack of relationship between preoperative ventricular size and either Rcsf or PVI is confirmed by our data. ${ }^{810}$ Even patients with a high Rcsf and low PVI did not have larger ventricles than those with less severely disturbed CSF dynamics. The magnitude of ventricular reduction after shunting too did not correlate with Rcsf or PVI.

The most important finding of this study was the relationship between rate of reduction in ventricular size and PVI. Patients demonstrating a rapid and marked reduction were found to have a significantly higher brain elasticity. Their Rcsf was higher as well but the difference with the patients of group 2, who presented slow and moderate to marked reduction in ventricular size, did not reach statistical significance. The mean Rcsf and PVI for the patients of group 2 were almost equal to those found for group 3 who exhibited slow and minimal to mild reduction to ventricle size. In group 2, however, CSF outflow resistance and brain elasticity were more or less inversely related, whereas in group 3 both CSF outflow resistance and brain elasticity tended to be either high or low. These results indicate that brain elasticity certainly is not the only factor responsible for ventricular reduction. CSF outflow resistance plays an additional role as well. In patients with high brain elasticity the presence of high Rcsf seems to increase the likelihood of a rapid and pronounced fall in ventricle size.

These inferences are valid for adult hydrocephalus only. In a group of hydrocephalic infants aged 1 week to 1 year the mean PVI was $28.1 \mathrm{ml}$, which is significantly higher than the $12.1 \mathrm{ml}$ predicted on the basis of the estimated volume of the neural axis for normal infants. ${ }^{9}$ On the other hand a very low mean PVI of $9.5 \mathrm{ml}$ was found in a series of adult patients with communicating hydrocephalus, half of whom had cerebral atrophy. ${ }^{10}$ Both the latter and our study included a few younger patients who exhibited a low PVI as well. The diametrical opposite findings for paediatric and adult hydrocephalus are difficult to ex- 
plain by differences in biomechanical properties of brain tissue alone. The more compliant skull of the infant, the cause, location and degree of the absorptive defect, the stage of the hydrocephalic process, differences in the methodology of PVI measurements and other unknown factors should be considered.

It can be concluded that single determinations of CSF outflow resistance and brain elasticity are not sufficient to explain satisfactorily the mechanism of ventricular dilatation and reduction. Therefore fundamental studies on the tissue properties of brain parenchyma, blood vessels, dura and skull, their relation to CSF parameters and how both are altered by the hydrocephalic process, are needed. ${ }^{18}$ In the clinical setting serial measurements of Rcsf and PVI, particularly during the developmental stage of hydrocephalus, would be revealing but are difficult to obtain. In the meantime we can deduce very little from this and our previous studies that support a more active role of brain elasticity in the pathogenesis of adult hydrocephalus. ${ }^{5611}$

We are grateful for financial support to the Van Leersumfonds, the Dr Ed. Hoelen Stichting and the Westeinde Researchfonds.

\section{References}

1 Shenkin HA, Greenberg JO, Grossman CB. Ventricular size after shunting for idiopathic normal pressure hydrocephalus. $J$ Neurol Neurosurg Psychiatry 1975;38:833-7.

2 Jacobs L, Kinkel WR, Painter F, Murawski J, Heffner Jr. RR. Computed tomography in dementia with special reference to changes in size of normal ventricles during aging and normal pressure hydrocephalus. In: Katzman R, Terry RD, Blick KL, eds. Alzheimer's Disease: Senile Dementia and Related Disorders. New York: Raven Press 1978:241-59.

3 Sprung C, Schultz B. Correlation of postoperative clinical course and ventricular size determined by computed tomography in normal pressure hydrocephalus. In: Driesen W, Brock M, Klinger M, eds. Advances in Neurosurgery 10, Computerized Tomography, Brain Metabolism, Spinal Injuries. Heidelberg: SpringerVerlag 1982:156-63.

4 Petersen RC, Mokri B, Laws ER. Surgical treatment of idiopathic hydrocephalus in elderly patients. Neurology 1985;35:307-11.
5 Tans JThJ, Poortvliet DCJ. Comparison of ventricular steady-state infusion with bolus infusion and pressure recording for differentiating between arrested and nonarrested hydrocephalus. Acta Neurochir 1984; 72:15-29.

6 Tans JThJ, Poortvliet DCJ. CSF outflow resistance and pressure-volume index determined by steady-state and bolus infusions. Clin Neurol Neurosurg 1985; 87:159-65.

7 Børgesen SE, Gjerris F. The predictive value of conductance to outflow of csf in normal pressure hydrocephalus. Brain 1982;105:65-86.

8 Børgesen SE, Gyldensted C, Gjerris F, Lester J. Computed tomography and pneumencephalography compared to conductance to outflow of csf in normal pressure hydrocephalus. Neuroradiology 1980; 20:17-22.

9 Shapiro K, Fried A, Marmarou A. Biomechanical and hydrodynamic characterization of the hydrocephalic infant. $J$ Neurosurg 1985;63:69-75.

10 Kosteljanetz M. CSF dynamics and pressure-volume relationships in communicating hydrocephalus. $J \mathrm{Neu}$ rosurg 1986;64:45-52.

11 Tans JThJ, Poortvliet DCJ. Steady-state and bolus infusions in hydrocephalus. In: Ishii S, Nagai H, Brock $\mathrm{M}$, eds. Intracranial Pressure $V$. Heidelberg, SpringerVerlag 1983:636-40.

12 Gomori JM, Steiner I, Melamed E, Cooper G. The assessment of changes in brain volume using combined linear measurements. Neuroradiology 1984;26:21-4.

13 Hirashima Y. Shindo K, Endo S. Measurement of the area of the anterior horn of the right lateral ventricle for the diagnosis of brain atrophy by CT. Neuroradiology 1983;25:23-7.

14 Meese W, Kluge W, Grumme T, Hopfenmüller W. CT evaluation of the csf spaces of healthy persons. Neuroradiology 1980;19:131-6.

15 McQuarrie IG, Saint-Louis L, Scherer PB. Treatment of normal pressure hydrocephalus with low versus medium pressure cerebrospinal fluid shunts. Neurosurgery 1984;15:484-8.

16 Ekstedt J, Friden H. Hydrodynamic properties of csf shunt systems. In: Shulman K, Marmarou A, Miller JD, Becker DP, Hochwald GM, Brock M, eds. Intracranial Pressure IV. Heidelberg, Springer-Verlag 1980:483-8.

17 Sainte-Rose C, Hooven MD, Hirsch JF. A new approach in the treatment of hydrocephalus. $J$ Neurosurg 1987;66:213-6.

18 Marmarou A. Biomechanics and theoretical models of hydrocephalus: Summary. In: Shapiro K, Marmarou A, Portnoy H, eds. Hydrocephalus. New York: Raven Press 1984:193-5. 